\title{
Feasibility of backscatter RFID systems on the human body
}

\author{
Jasmin Grosinger
}

\begin{abstract}
In this contribution, the author examines the feasibility of on-body backscatter radio frequency identification (RFID) systems in the ultra high frequency range. Four different on-body RFID systems are investigated operating monopoles or patch antennas at $900 \mathrm{MHz}$ or $2.45 \mathrm{GHz}$. The systems' feasibility is analyzed by means of on-body channel measurements in a realistic test environment. The measured channel transfer functions allow to evaluate if enough power is available for a reliable backscatter communication. This evaluation is done with the aid of outage probabilities in the forward link and the backward link of the systems. Using these probabilities, the on-body systems prove feasible when using state-of-the-art reader and transponder chips. In particular, the use of semi-passive RFID transponder chips leads to a reliable performance in the systems' forward links. The robust performance of the systems' backward links is clearly shown for the $900 \mathrm{MHz}$ monopole antenna configuration, while the limitations in the backward links of the other systems have to be overcome by the use of a second reader unit on the person's back. The novel feasibility analysis presented here allows to examine each system parameter individually and thus leads to reliable and robust backscatter RFID systems.
\end{abstract}

Keywords: Wireless body area networks, Backscatter Radio Frequency Identification (RFID) systems, On-body RFID systems, RFID outage probabilities, On-body channel measurements, Feasibility analysis

\section{Introduction}

Wireless body area networks (WBANs) enable many new promising applications in the field of remote health monitoring, therapy support at home, wellness, and fitness. Therefore, the attention of the industry and the scientific community is highly drawn to WBANs [1,2]. WBANs connect sensor nodes situated in clothes, on the body, or under the skin of a person through a wireless communication channel.

A promising communication technology for WBANs is backscatter radio frequency identification (RFID) in the ultra high frequency (UHF) range. Backscatter RFID relies on the radio communication between an RFID reader, acting as a control unit, and a multitude of passive or semi-passive RFID transponders (tags), acting as sensor nodes. The principle of communication for transmitting information from the tag to the reader relies on a modulated backscatter signal. All power for the transmission

Correspondence: jasmin.grosinger@gmail.com

Vienna University of Technology, Institute of Telecommunications,

Gusshausstrasse 25/389, 1040 Vienna, Austria of the sensor data is drawn from the electromagnetic field radiated by the reader. Hence, their low-power consumption makes backscatter tags appropriate for WBANs that require small, light-weight, and low-maintenance sensor nodes. In addition, research efforts are ongoing to integrate sensing capabilities in backscatter tags without further enhancing their power consumption [3-5]. Such sensor tags can then be beneficially used to monitor the physiological parameters of a person (e.g., blood pressure, temperature, heartbeat, or body motion).

In backscatter RFID systems, it is vital to ensure a reliable power transmission to the backscatter tags and to realize a robust wireless communication between the reader and the tags [6]. To assure this, it is advisable to investigate the on-body radio propagation channel including the effects of the antennas and to study the wireless power transmission and communication in realistic operating environments. These characteristics are investigated in this contribution which studies the feasibility of four different backscatter RFID systems in a WBAN.

\section{望 Springer}

(c) 2013 Grosinger; licensee Springer. This is an Open Access article distributed under the terms of the Creative Commons Attribution License (http://creativecommons.org/licenses/by/2.0), which permits unrestricted use, distribution, and reproduction in any medium, provided the original work is properly cited. 
Previous studies on UHF RFID-based WBANs have focused on in-body [7-9] and off-body [10-12] communication systems. So far, the investigation of backscatter communication systems on the human body has received less attention in the literature. A first feasibility analysis of an on-body backscatter RFID system is presented in [13] and is based on indoor backscatter measurements at $870 \mathrm{MHz}$. The investigated RFID system consisted of an on-body reader with a patch antenna and five on-body tags composed of custom-built wearable felt antennas.

This contribution provides a feasibility analysis of four different on-body RFID systems. The feasibility analysis is based on outage probabilities derived from on-body channel measurements in a realistic test environment at $900 \mathrm{MHz}$ and $2.45 \mathrm{GHz}$. In comparison to backscatter measurements, the analysis based on channel measurements allows to examine each system parameter individually and thus gives a deeper insight in the wireless power transmission and communication of backscatter RFID systems. The investigated RFID systems are composed of custom-built monopole or patch antennas operating at $900 \mathrm{MHz}$ or $2.45 \mathrm{GHz}$. Each antenna acts as both reader and tag antennas.

The article is organized as follows. Section 2 describes the investigated on-body RFID systems and defines outage probabilities for a backscatter RFID system. The outage probabilities of the investigated RFID systems are then found by means of on-body channel measurements in Section 3. Section 4 analyzes the feasibility of the different on-body RFID systems based on these measurements.

\section{On-body RFID systems}

This contribution provides an investigation of four different on-body backscatter RFID systems. Figure 1 shows the arrangement of the on-body RFID reader and sensor tags situated on the body of an adult female. The RFID reader is situated on the stomach of the female, while four RFID tags are placed at various positions on the female's body: on the right chest, on the middle of the back, on the left side of the head, and on the right wrist. This arrangement leads to four typical on-body links which represent two trunk-to-trunk, a trunk-to-head, and a trunk-to-limb links following a classification of on-body links as introduced in [14].

The investigated RFID systems are composed of custom-built monopole or patch antennas operating at $900 \mathrm{MHz}$ or $2.45 \mathrm{GHz}$ (see Figure 2). Each antenna acts as both reader and tag antennas. For an adequate antenna design, a human body model has been created in Ansys' HFSS (high frequency structure simulator) [15]. The simplified model is an appropriate tool to design on-body antennas $[16,17]$.

Practically, monopole antennas are not suitable for WBAN applications because they are not low profile.

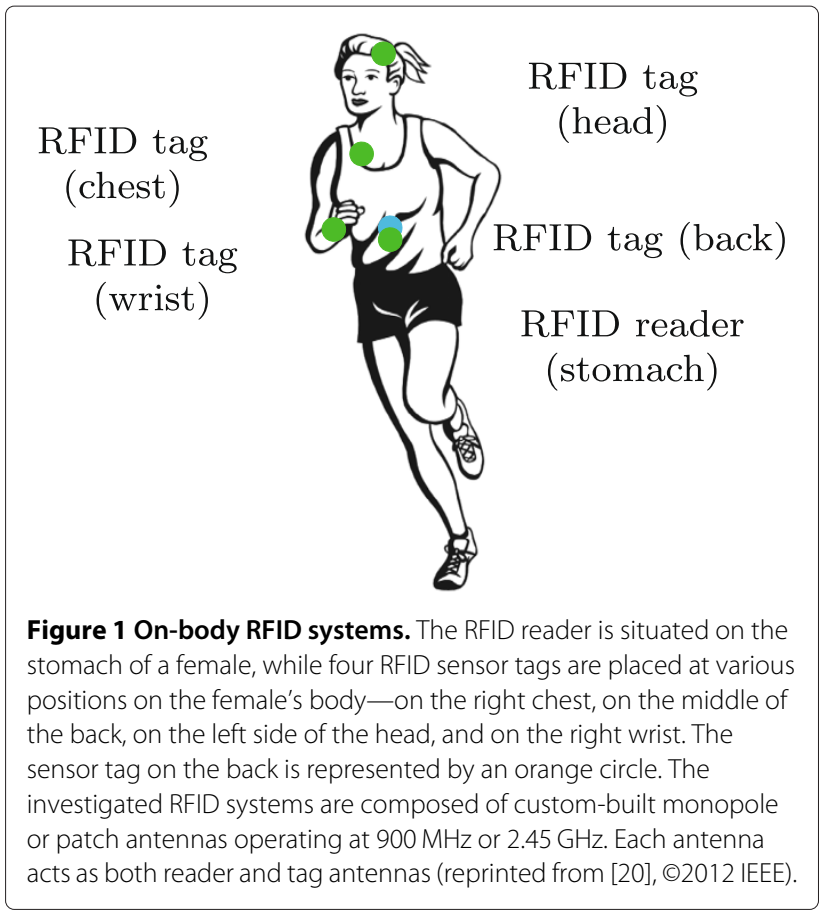

However, monopoles show the best performance in onbody systems $[18,19]$ and are used as a best-case reference in this study to define an upper bound for the performance of practical system implementations. Conversely, low profile patch antennas are especially suitable for onbody applications [18]. In this study, the less efficient patch antennas provide an insight in the performance of practical system implementations [20].

\subsection{RFID outage probabilities}

In backscatter RFID systems, a bidirectional radio link is established between the reader and the tag-the readertag-reader link-which can be subclassified into a forward link and a backward link. Figure 3 outlines the link budget of a backscatter radio system.

In the forward link, the reader transmits radio frequency (RF) power, $P_{\mathrm{TX}, \text { Reader, }}$ and data to the tag. The RFID tag consists of an antenna and a microchip. The power absorbed by the chip, $P_{\text {Chip }}$, is defined as

$$
P_{\text {Chip }}=\tau P_{\text {Tag }}=\tau\left|S_{21}\right|^{2} P_{\mathrm{TX}, \text { Reader }},
$$

where $\tau$ is the power transmission coefficient between the tag's antenna and chip [17] and $P_{\text {Tag }}$ is the power received by the tag's antenna. $S_{21}$ is the channel transfer function between the reader antenna and the tag antenna, $\left|S_{21}\right|^{2}$ defines the channel gain in the forward link of the system.

If $P_{\text {Chip }}$ is smaller than the minimum power which is required to activate the chip-which is denoted by the chip's sensitivity, $T_{\text {Chip }}$,-the backscatter communication 


\section{Monopole antennas}
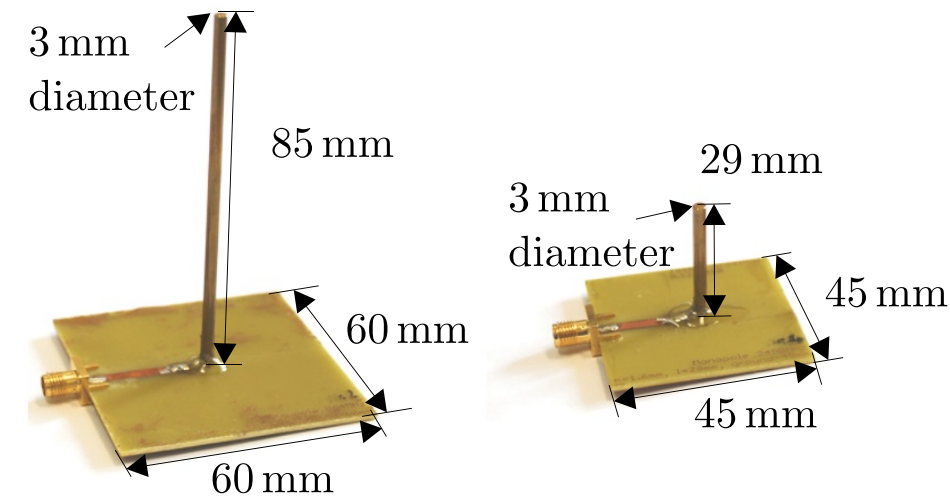

\section{Patch antennas}
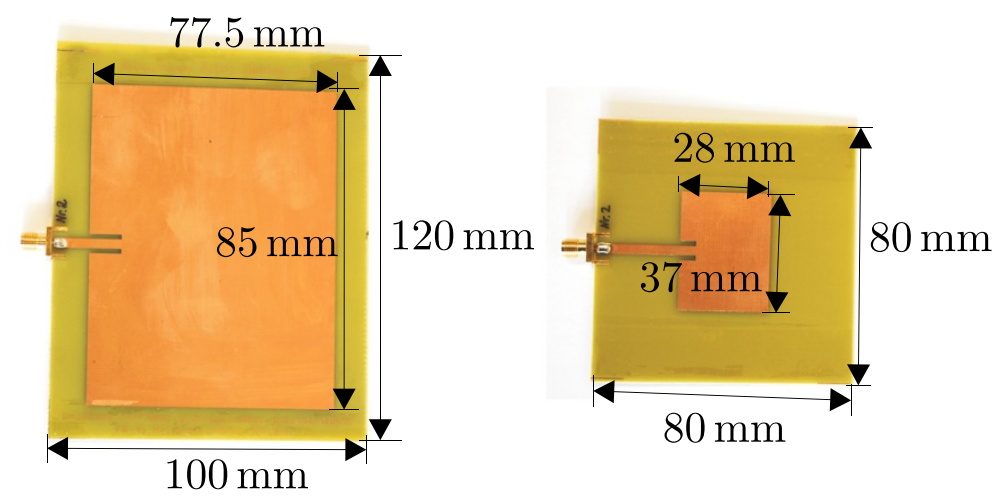

Figure 2 On-body antennas. On-body monopole and patch antennas resonant at $900 \mathrm{MHz}$ (left) and $2.45 \mathrm{GHz}$ (right): The antennas were designed by means of a human body model and realized on FR-4 substrate with a thickness of $1.6 \mathrm{~mm}$. The designs were optimized for an antenna-to-body separation distance of $5 \mathrm{~mm}$. Details about the antenna properties can be found in [20] (reprinted from [20], O2012 IEEE).

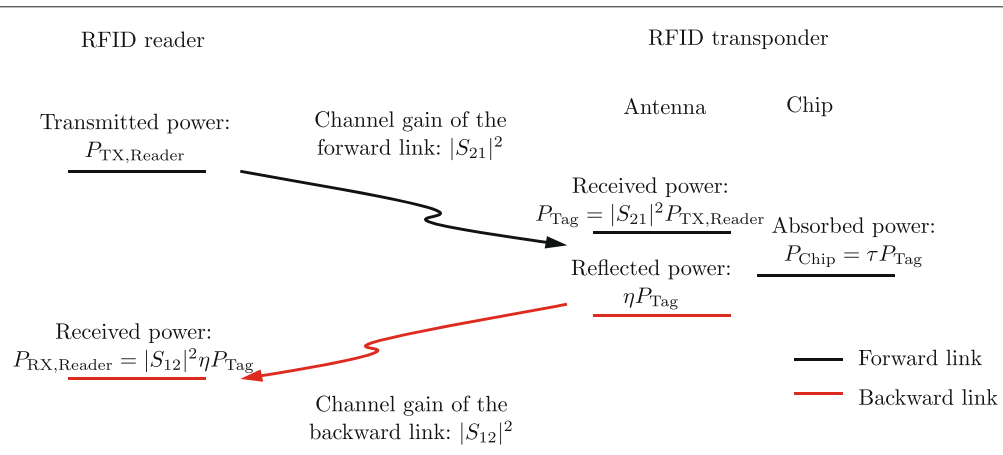

Figure 3 Link budget of a backscatter RFID system. Link budget of a backscatter RFID system: In the forward link, the reader transmits RF power and data to the tag. The RFID tag consists of an antenna and a microchip. The power received by the tag antenna is $P_{\text {Tag }}=\left|S_{21}\right|^{2} P_{\text {TX, Reader }}$. The power absorbed by the chip is $P_{\text {Chip }}=\tau P_{\text {Tag, }}$, where $\tau$ is the power transmission coefficient of the tag. In the backward link, the tag responds to the reader by modulating the backscattered signal. The received power at the reader can be written as $P_{\mathrm{RX}, \text { Reader }}=\left|S_{12}\right|^{2} \eta P_{\text {Tag }}, \eta$ is the modulation efficiency of the tag. 


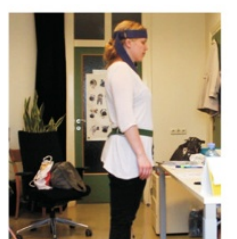

Standing, upright

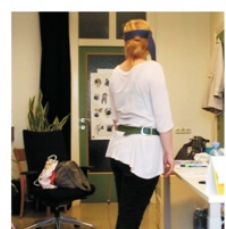

Standing, body turned left

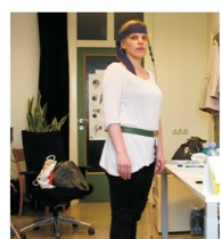

Standing, body turned right

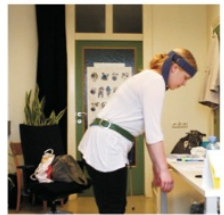

Standing, body leaning forward

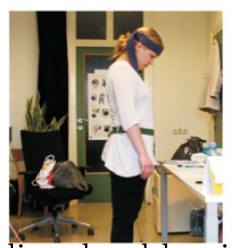

Standing, head leaning

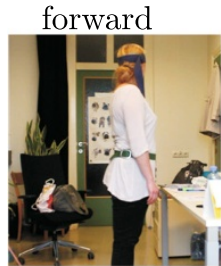

Standing, head turned left

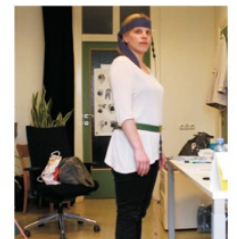

Standing, head turned

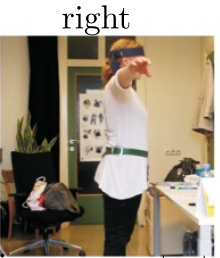

Standing, arms stretched out to sides

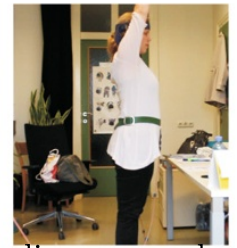

Standing, arms above

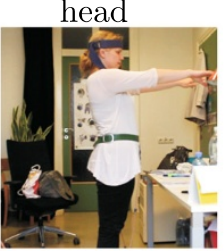

Standing, arms reaching forward

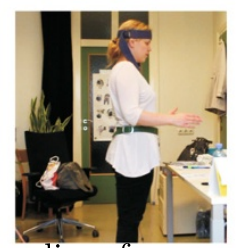

Standing, forearms

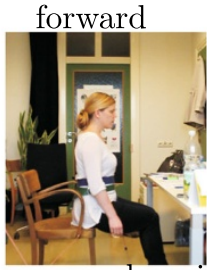

Sitting, arms hanging body

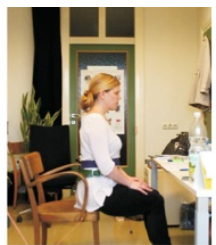

Sitting, hands in lap

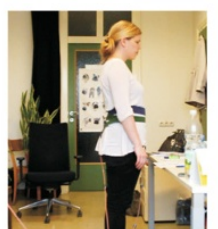

Standing, upright

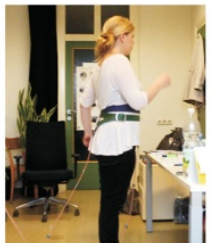

Standing, moving arms, head, and body randomly

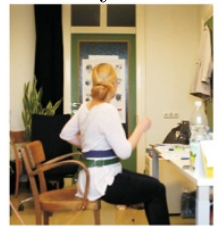

Sitting, moving arms, head, and body randomly

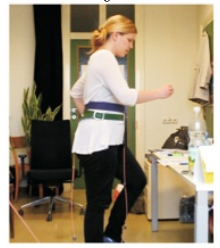

Walking, back and forth

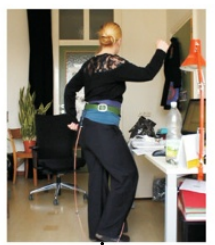

Walking, moving arms, head, and body randomly

Figure 4 Snapshot of different body postures. Snapshots of body postures performed during the on-body channel measurements: The channel transfer functions, $S_{21}$ and $S_{12}$, of all four on-body RFID systems were measured versus 18 different stationary and moving body postures in an indoor multipath environment. The snapshots were taken during several measurement runs of the stomach-head and stomach-back links. 


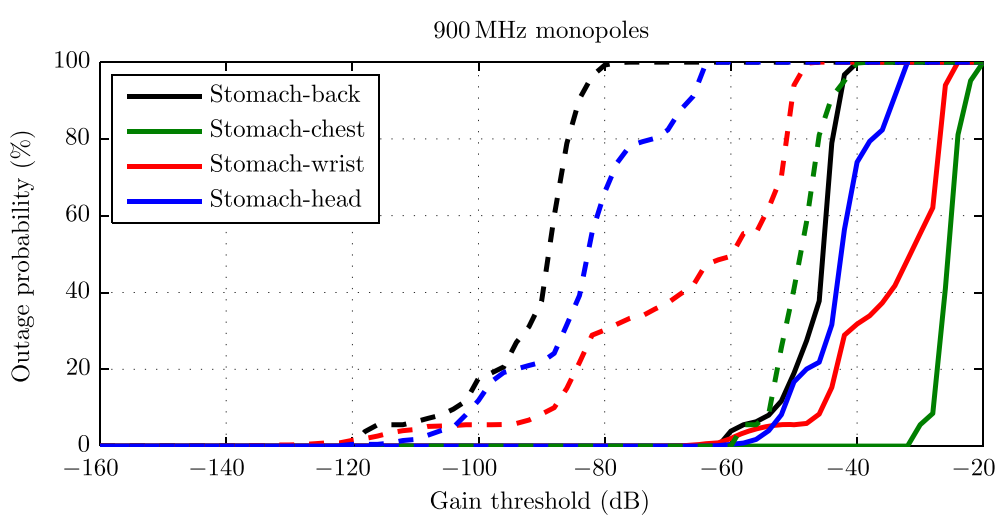

Figure 5 Outage probabilities of $\mathbf{9 0 0} \mathbf{~ M H z}$ monopoles. Outage probabilities of the on-body RFID system operating the $900 \mathrm{MHz}$ monopoles: The outage probabilities of the forward link and the backward link are plotted for all four on-body links versus their respective gain thresholds (solid lines: forward link, dashed lines: backward link). The outage probability of the forward link can be found by plotting the CDF of the measured channel gain of the forward link, $P_{F}=P\left\{\left|S_{21}\right|^{2} \leq F_{T h}\right\}$. The gain threshold of the forward link, $F_{T h}$, is defined as the minimum channel gain that is necessary to initiate the chip's data transmission. The outage probability of the backward link can be found by plotting the CDF of the product of the measured channel gains in forward and backward links, $P_{\mathrm{B}}=\mathrm{P}\left\{\left|S_{21}\right|^{2}\left|S_{12}\right|^{2} \leq B_{\mathrm{Th}}\right\}$. The gain threshold of the backward link, $B_{T h}$, is defined as the minimum channel gain of the forward and backward links that is necessary to correctly receive the tag's data.

is limited in its forward link [6]. The minimum channel gain, $\left|S_{21}\right|^{2}$, that is necessary to avoid such a limitation, defines a gain threshold for the system's forward link (see Equation (1), $P_{\text {Chip }}=T_{\text {Chip }}$ ),

$$
F_{\mathrm{Th}}=\frac{T_{\text {Chip }}}{\tau P_{\mathrm{TX}, \text { Reader }}} .
$$

The probability that the channel gain is equal or smaller than this gain threshold is

$$
P_{\mathrm{F}}=\mathrm{P}\left\{\left|S_{21}\right|^{2} \leq F_{\mathrm{Th}}\right\}
$$

and defines the outage probability of the system's forward link, more precisely the probability that the backscatter system operates at its limit. This outage probability can be found by plotting the cumulative distribution function
(CDF) of the measured channel gain in the forward link, $\left|S_{21}\right|^{2}[21]$.

In the backward link, the tag responds to the reader by modulating the backscattered signal [22]. The power of the tag's signal at the receiver (RX) of the reader, $P_{\mathrm{RX}, \text { Reader, }}$ can be written as

$$
P_{\text {RX,Reader }}=\left|S_{12}\right|^{2} \eta P_{\text {Tag }}=\left|S_{12}\right|^{2} \eta\left|S_{21}\right|^{2} P_{\text {TX,Reader }},
$$

where $\eta$ is the modulation efficiency of the tag [17]. $S_{12}$ is the channel transfer function between the tag antenna and the reader antenna. $\left|S_{12}\right|^{2}$ defines the channel gain in the backward link.

$P_{\mathrm{RX}, \text { Reader }}$ should be equal or bigger than the RX's sensitivity, $T_{\mathrm{RX} \text {,Reader }}$, that is defined as the minimum input power at the reader to assure a successful reception of the tag's data. If $P_{\mathrm{RX}, \text { Reader }}$ is smaller than $T_{\mathrm{RX} \text {, Reader, }}$, the

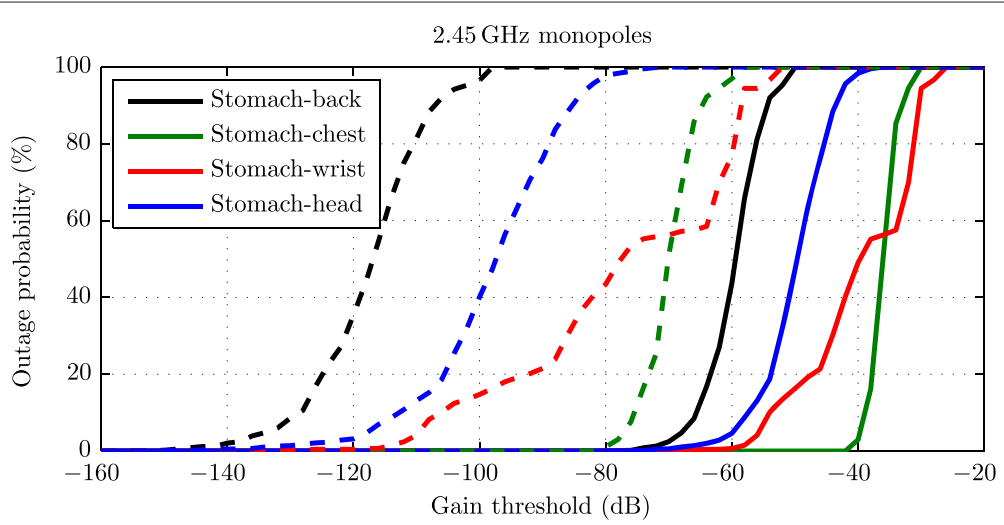

Figure 6 Outage probabilities of $2.45 \mathrm{GHz}$ monopoles. Outage probabilities of the on-body RFID system operating the $2.45 \mathrm{GHz}$ monopoles: The outage probabilities of the forward link and the backward link are plotted for all four on-body links versus their respective gain thresholds (solid lines: forward link, dashed lines: backward link). 


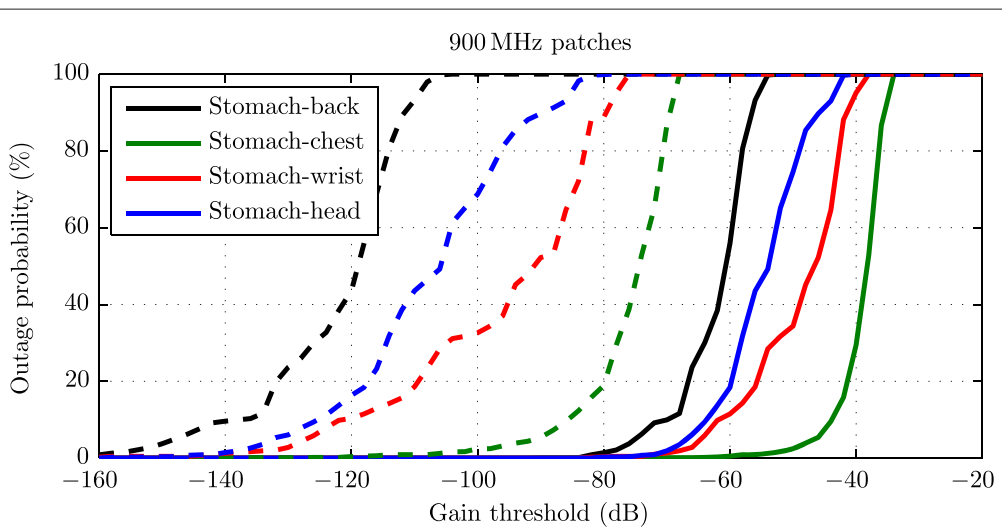

Figure 7 Outage probabilities of $900 \mathbf{~ M H z ~ p a t c h e s . ~ O u t a g e ~ p r o b a b i l i t i e s ~ o f ~ t h e ~ o n - b o d y ~ R F I D ~ s y s t e m ~ o p e r a t i n g ~ t h e ~} 900 \mathrm{MHz}$ patches: The outage probabilities of the forward link and the backward link are plotted for all four on-body links versus their respective gain thresholds (solid lines: forward link, dashed lines: backward link).

communication system is limited in its backward link [6]. The total minimum channel gain, $\left|S_{21}\right|^{2}\left|S_{12}\right|^{2}$, that is necessary to realize $P_{\mathrm{RX}, \text { Reader }}=T_{\mathrm{RX}, \text { Reader }}$, characterizes a gain threshold for the backward link of the system (see Equation (4)),

$$
B_{\mathrm{Th}}=\frac{T_{\mathrm{RX}, \text { Reader }}}{\eta P_{\mathrm{TX}, \text { Reader }}} .
$$

The outage probability of the system's backward link is defined as the probability that the total channel gain is equal or smaller than this gain threshold,

$$
P_{\mathrm{B}}=\mathrm{P}\left\{\left|S_{21}\right|^{2}\left|S_{12}\right|^{2} \leq B_{\mathrm{Th}}\right\} .
$$

This probability can be found by plotting the CDF of the product of the measured channel gain in the forward link and the backward link, $\left|S_{21}\right|^{2}\left|S_{12}\right|^{2}$.

\section{On-body channel measurements}

To examine the outage probabilities of the on-body backscatter systems, the channel transfer functions, $S_{21}$ and $S_{12}$, were measured for all four antenna configurations by means of a vector network analyzer $[16,17]$. The channel transfer functions depend on the antenna characteristics of the reader and the tag (e.g., antenna gain and polarization) and on the properties of the on-body radio links (e.g., path length and fading). The functions were measured in a realistic test scenario versus 18 different stationary and moving body postures in an indoor multipath environment. Figure 4 shows snapshots of the different postures that are based on a list of body postures published in [14]. Each posture was held $20 \mathrm{~s}$, the repetition rate of the measurement was $5 \mathrm{~s}^{-1}$. As a result, the channel transfer functions are composed of 1800 measurement points at the specific frequency.

The measurement results are plotted in Figures 5, 6, 7, and 8 for all four antenna configurations. Each figure plots

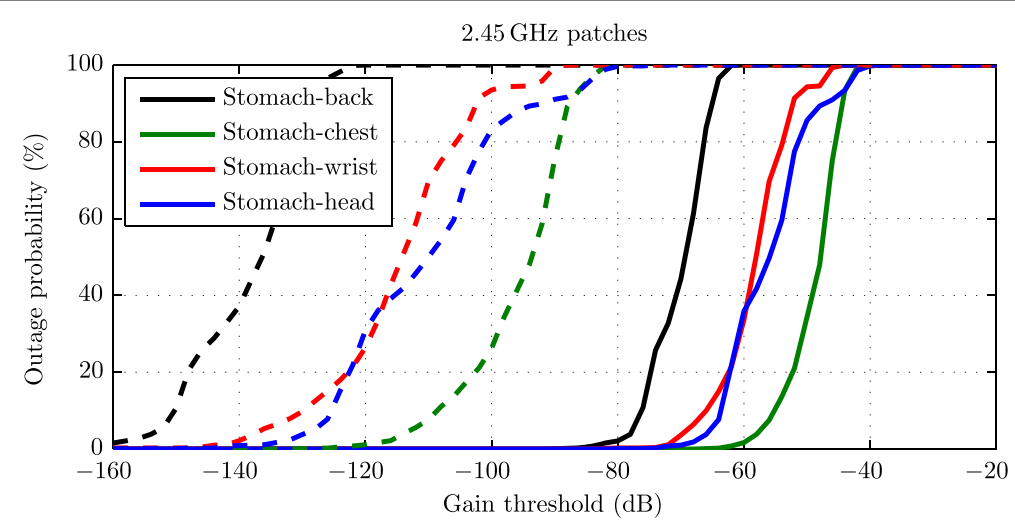

Figure 8 Outage probabilities of $2.45 \mathrm{GHz}$ patches. Outage probabilities of the on-body RFID system operating the $2.45 \mathrm{GHz}$ patches: The outage probabilities of the forward link and the backward link are plotted for all four on-body links versus their respective gain thresholds (solid lines: forward link, dashed lines: backward link). 
the outage probabilities of the forward and backward links versus their respective gain thresholds. As introduced in the previous section, the outage probability of the forward link is found by plotting the CDF of the measured channel gain, $\left|S_{21}\right|^{2}$, according to Equation (3). The outage probability of the backward link is found by plotting the CDF of the product of the measured channel gains in the forward link and the backward link, $\left|S_{21}\right|^{2}\left|S_{12}\right|^{2}$, according to Equation (6). Figures 5 and 6 show the outage probabilities of the RFID systems operating the $900 \mathrm{MHz}$ and $2.45 \mathrm{GHz}$ monopole antennas, while Figures 7 and 8 plot the results for the $900 \mathrm{MHz}$ and $2.45 \mathrm{GHz}$ patch antenna configurations.

\section{Feasibility analysis}

In the following, the different on-body RFID systems are analyzed by means of the outage probabilities found in the previous section.

As expected from theory and from previous measurement campaigns at $2.45 \mathrm{GHz}$ [14], Figure 5 shows that on-body links with longer path lengths have higher outage probabilities in comparison to links with shorter distances (compare, e.g., the stomach-back link with the stomachchest link). In addition, the link geometry and thus the channel gain are influenced by the movements of the body.
The strength of this influence depends on the on-body link. An on-body link with a higher mobility experiences a wider range of outage probabilities than trunk-to-trunk links with lower mobility (compare, e.g., the stomachwrist link with the stomach-chest link). These behaviors can be observed for all four antenna configurations in their forward and backward links (see Figures 6, 7 and 8).

In addition, the figures show that the outage probabilities of the $900 \mathrm{MHz}$ antennas are lower than the probabilities of the $2.45 \mathrm{GHz}$ antennas (compare, e.g., Figures 5 and 6). This difference is due to an increased energy absorption in human tissues at higher frequencies [23]. Again, this behavior can be observed for both antenna types, the monopoles and the patch antennas.

Furthermore, the probability curves of all four figures show that the monopoles act indeed as best-case references.

\subsection{State-of-the-art example}

Subsequently, the outage probabilities for all four antenna configurations are explored individually for each system parameter of a state-of-the-art system example, i.e., $P_{\mathrm{TX}, \text { Reader }}, \tau, T_{\text {Chip }}, \eta$, and $T_{\mathrm{RX} \text {,Reader. }}$ In general, the maximum permitted outage probabilities are governed
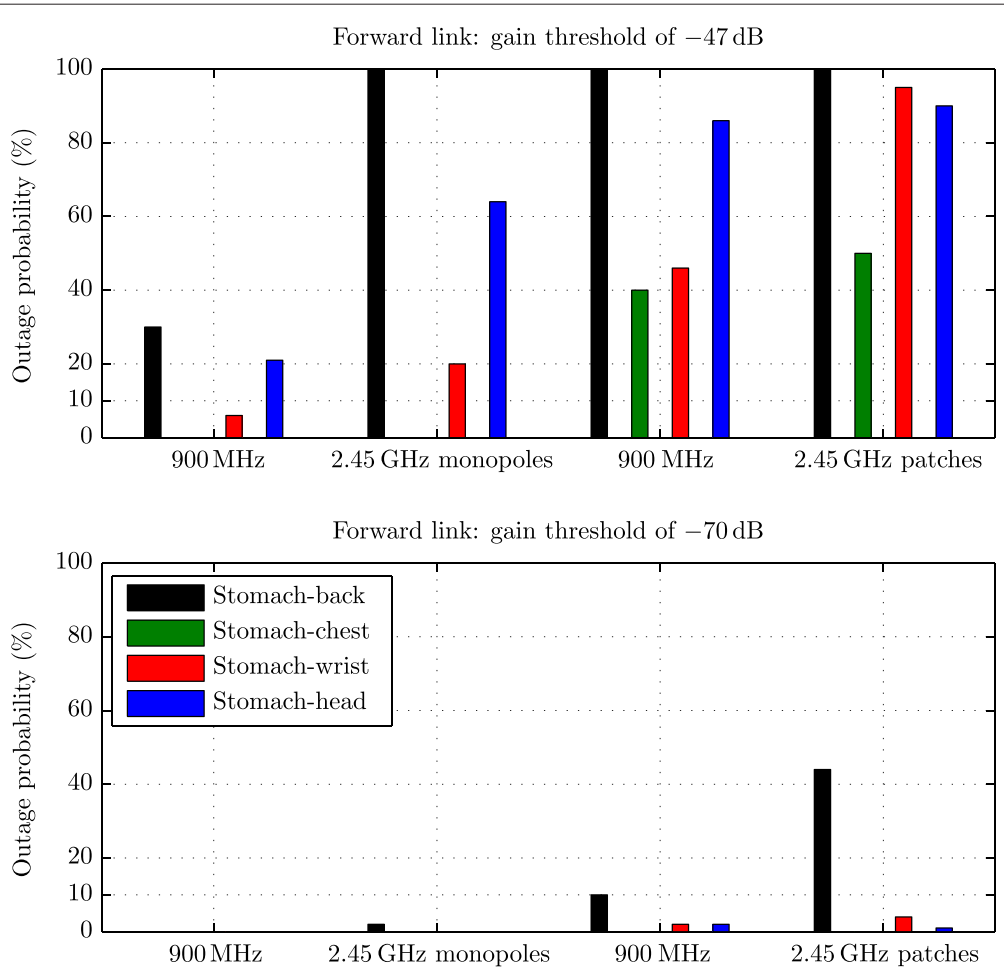

Figure 9 Outage probabilities for $\boldsymbol{F}_{\mathbf{T h}}=\mathbf{- 4 7} \mathrm{dB}$ and $\boldsymbol{F}_{\mathbf{T h}}=-\mathbf{7 0} \mathrm{dB}$. Outage probabilities for two specific system implementations which lead to gain thresholds of $F_{\mathrm{Th}}=-47 \mathrm{~dB}$ and $F_{\mathrm{Th}}=-70 \mathrm{~dB}$ (upper bar chart: $-47 \mathrm{~dB}$, lower bar chart: $-70 \mathrm{~dB}$ ): The outage probabilities of the forward link are plotted for all four on-body links and for all four antenna configurations ( $900 \mathrm{MHz}$ and $2.45 \mathrm{GHz}$ monopoles, $900 \mathrm{MHz}$ and $2.45 \mathrm{GHz}$ patches). The allowed outage probabilities are 10\% for all on-body RFID system implementations. 
by the application. The outage probabilities should be close to zero for a system that monitors life parameters of patients in clinical care, while systems used in sports analysis can deal with higher outage probabilities. In the following, the systems allow a maximum outage probability of $10 \%$.

In the state-of-the-art system, the on-body reader consists of an Impinj Indy reader chip with an external power amplifier [24]. The reader provides a maximum transmit power of $P_{\mathrm{TX} \text {,Reader }}=30 \mathrm{dBm}$ and an RX sensitivity of $T_{\mathrm{RX}, \text { Reader }}=-95 \mathrm{dBm}$. The on-body tags consist of passive Monza 5 tag chips [25]. The passive chips provide a sensitivity of $T_{\text {Chip }}=-17.8 \mathrm{dBm}$. The power transmission coefficient is assumed to be $100 \%$, while the modulation efficiency is $20 \%$. These system parameters lead to gain thresholds of $F_{\mathrm{Th}}=-47 \mathrm{~dB}$ and $B_{\mathrm{Th}}=-118 \mathrm{~dB}$ (see Equations (2) and (5)).

The upper bar chart of Figure 9 shows the outage probabilities of the forward link for the gain threshold of $F_{\mathrm{Th}}=-47 \mathrm{~dB}$. The figure plots the probabilities for each antenna configuration and for each on-body link. The probabilities are deduced from Figures 5, 6, 7, and 8 . Figure 9 shows that the investigated RFID systems are considerably limited in their forward link, i.e. the outage probabilities are above $10 \%$. There are different strategies to overcome these limitations. An increase in the transmit power is not an option because of the safety regulations and power constraints in on-body systems [26,27]. Another strategy is to use semi-passive backscatter tags with chip sensitivities down to $-40 \mathrm{dBm}$. Such a sensitivity leads to a gain threshold in the forward link of $F_{\mathrm{Th}}=-70 \mathrm{~dB}$. The lower bar chart of Figure 9 shows that semi-passive tags lead to a good performance in the on-body systems' forward links, i.e., to outage probabilities lower than $10 \%$. However, there is still a rather large limitation in the stomach-back link of the $2.45 \mathrm{GHz}$ patch antenna system. This constraint can be resolved by the use of more efficient patch antennas realized on a low-loss substrate or by the use of higher mode patches which benefit surface waves along the human body like monopole antennas. Another solution would be the use of a second RFID reader on the female's back to reduce the path lengths.

The upper bar chart of Figure 10 shows the outage probabilities in the systems' backward links for a gain threshold of $B_{\mathrm{Th}}=-118 \mathrm{~dB}$. Limitations can be observed in the on-body systems operating the $2.45 \mathrm{GHz}$ monopoles, the $900 \mathrm{MHz}$ and $2.45 \mathrm{GHz}$
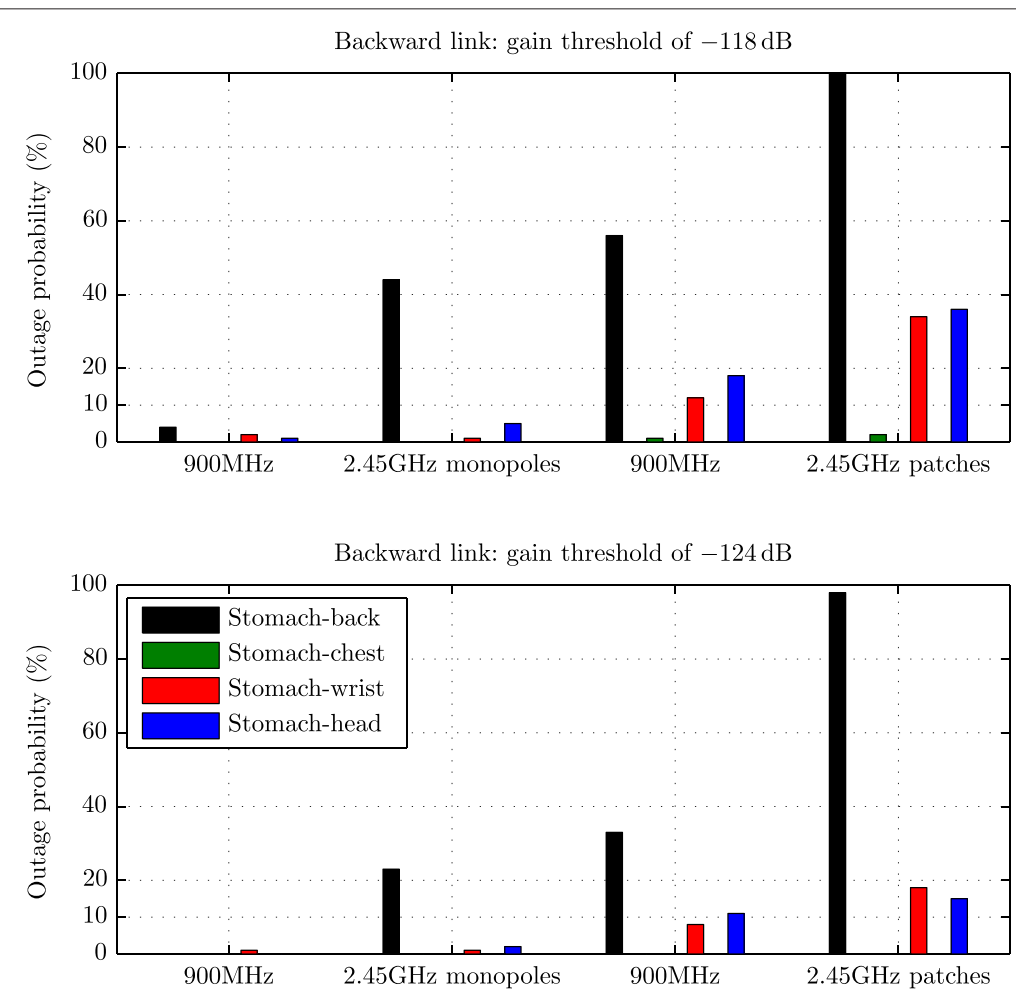

Figure 10 Outage probabilities for $\boldsymbol{B}_{\mathrm{Th}}=\mathbf{- 1 1 8} \mathrm{dB}$ and $\boldsymbol{B}_{\mathbf{T h}}=\mathbf{- 1 2 4} \mathrm{dB}$. Outage probabilities for two specific system implementations which lead to gain thresholds of $B_{T h}=-118 \mathrm{~dB}$ and $B_{T h}=-124 \mathrm{~dB}$ (upper bar chart: $-118 \mathrm{~dB}$, lower bar chart: $-124 \mathrm{~dB}$ ): The outage probabilities of the backward link are plotted for all four on-body links and for all four antenna configurations ( $900 \mathrm{MHz}$ and $2.45 \mathrm{GHz}$ monopoles, $900 \mathrm{MHz}$ and $2.45 \mathrm{GHz}$ patches). The allowed outage probabilities are 10\% for all on-body RFID system implementations. 
patches. To overcome these limitations, a phasemodulated backscatter signal can be used [17]. This modulation scheme provides a maximum modulation efficiency of $\eta=81 \%$ and leads to gain threshold of about $-124 \mathrm{~dB}$. However, if a phase-modulated backscatter signal is applied, there should be no substantial limitations in the system's forward link [17]. The lower bar chart of Figure 10 shows the outage probabilities in the backward link for the $-124 \mathrm{~dB}$ threshold. The $6 \mathrm{~dB}$ difference in the threshold only slightly improves the performance of the systems. Further strategies have to be applied to overcome all limitations in the backward links, in particular in the stomach-back links. A promising strategy is to use a second reader unit on the female's back, other strategies are e.g., the realization of more sophisticated antennas or the use of a reader RX with a lower sensitivity.

\section{Conclusions}

In conclusion, the author demonstrated the feasibility of backscatter UHF RFID systems on the human body at $900 \mathrm{MHz}$ and $2.45 \mathrm{GHz}$. Until now, the investigation of on-body backscatter RFID systems has received less attention in the literature. A first feasibility analysis of an on-body backscatter RFID system was based on indoor backscatter measurements at $870 \mathrm{MHz}$, where the investigated RFID system consisted of an on-body reader with a patch antenna and on-body tags composed of custombuilt wearable felt antennas. In this contribution, four different on-body RFID systems are investigated operating two different types of on-body antennas. Monopole antennas act as best-case references, while the less efficient patch antennas are used to give an insight into practical RFID system implementations.

In this article, the author presents a novel feasibility analysis based on on-body channel measurements in a realistic test environment. In particular, the channel transfer functions of the systems were measured versus different stationary and moving body postures and led to outage probabilities of the systems' forward and backward links. These outage probabilities helped to easily identify limitations in the backscatter systems and to evaluate strategies to overcome these barriers for the realization of reliable on-body RFID systems. In comparison to backscatter measurements, the analysis based on channel measurements allows to examine each system parameter individually and thus gives a deeper insight in the wireless power transmission and communication of backscatter RFID systems.

It is worth pointing out that the presented analysis can be performed for any kind of backscatter RFID system. The analysis provides an initial overview of a backscatter system and ultimately allows to realize a reliable power transmission to the chips and a robust wireless communication between the reader and the tags.

\section{Competing interests}

The author declares that she has no competing interests.

\section{Acknowledgements}

This study was performed as part of the project "MAS-Nanoelectronics for Mobile Ambient Assisted Living-Systems" which is funded by "ENIAC Joint Undertaking" and Austria's "Österreichische

Forschungsförderungsgesellschaft".

\section{Received: 30 December 2012 Accepted: 25 February 2013}

Published: 20 March 2013

\section{References}

1. Y Hao, R Foster, Wireless body sensor networks for health-monitoring applications. Physiol. Meas. 29(11), R27-R56 (2008)

2. S Patel, H Park, P Bonato, L Chan, M Rodgers, A review of wearable sensors and systems with application in rehabilitation. J. NeuroEng. Rehab. 9(21), $1-17(2012)$

3. A Sample, D Yaeger, P Powledge, A Mamishev, J Smith, Design of an RFID-based battery-free programmable sensing platform. IEEE Trans. Instrum. Meas. 57(11), 2608-2615 (2008)

4. G Marrocco, Multiport sensor RFIDs for wireless passive sensing of objects-basic theory and early results. IEEE Trans. Antennas Propag. 56(8), 2691-2702 (2008)

5. J Grosinger, J Griffin, in Proc. IEEE Antennas and Propagation Society International Symposium. A bend transducer for backscatter RFID sensors, (2012), doi:10.1109/APS.2012.6349258

6. P Nikitin, K Rao, in Proc IEEE Antennas and Propagation Society International Symposium. Performance limitations of passive UHF RFID systems, (2006), pp. 1011-1014, doi:10.1109/APS.2006.1710704

7. A Sani, M Rajab, R Foster, Y Hao, Antennas and propagation of implanted RFIDs for pervasive healthcare applications. Proc. IEEE. 98(9), 1648-1655 (2010)

8. C Schmidt, D Valderas, J Garcia, I Ortego, X Chen, in Proc. European Conference on Antennas and Propagation. Passive UHF RFID near field link budget for implanted sensors, (2011), pp. 3479-3483

9. C Occhiuzzi, G Contri, G Marrocco, Design of implanted RFID tags for passive sensing of human body: the STENTag. IEEE Trans. Antennas Propag. 60(7), 3146-3154 (2012)

10. M Polivka, M Svanda, P Hudec, S Zvanovec, UHF RF identification of people in indoor and open areas. IEEE Trans. Microw. Theory Tech. 57(5), 1341-1347 (2009)

11. C Occhiuzzi, S Cippitelli, G Marrocco, Modeling, design and experimentation of wearable RFID sensor tag. IEEE Trans. Antennas Propag. 58(8), 2490-2498 (2010)

12. S Cotton, W Cully, W Scanlon, J McQuiston, in Proc. Loughborough Antennas and Propagation Conference. Channel characterisation for indoor wearable active RFID at $868 \mathrm{MHz}$, (2011), pp. 1-4, doi:10.1109/LAPC.2011.6114085

13. S Manzari, C Occhiuzzi, G Marrocco, Feasibility of body-centric systems using passive textile RFID Tags. IEEE Antennas Propag. Mag. 54(4), 49-62 (2012)

14. P Hall, Y Hao, Antennas and Propagation for Body-Centric Wireless Communications. (Artech House Inc., USA, 2006)

15. ANSYS Inc ANSYS HFSS (2012). [http://www.ansys.com/Products/ Simulation+Technology/Electromagnetics/High-Performance+ Electronic+Design/ANSYS+HFSS]

16. J Grosinger, M Fischer, in Proc. IEEE-APS Topical Conference on Antennas and Propagation in Wireless Communications. Indoor on-body channel measurements at $900 \mathrm{MHz},(2011), \mathrm{pp} .1037-1040$, doi:10.1109/APWC.2011.6046824

17. J Grosinger, Backscatter radio frequency systems and devices for novel wireless sensing applications (2012). Ph.d. thesis, Vienna University of Technology

18. G Conway, W Scalon, Antennas for over-body-surface communication at 2.45 GHz. IEEE Trans. Antennas Propag. 57(4), 844-855 (2009)

19. P Hall, Y Hao, Y Nechayev, A Alomainy, C Constantinou, C Parini, M Kamarudin, T Salim, D Hee, R Dubrovka, A Owadally, W Song, A Serra, P Nepa, M Gallo, M Bozzetti, Antennas and propagation for on-body communication systems. IEEE Antennas Propag. Mag. 49(3), 41-58 (2007) 
20. J Grosinger, M Fischer, in Proc. International EURASIP Workshop on RFID Technology. Evaluating on-body RFID systems at $900 \mathrm{MHz}$ and $2.45 \mathrm{GHz}$, (2012), pp. 52-58, doi:10.1109/RFID.2012.14

21. G Lasser, R Langwieser, F Xaver, C Mecklenbräuker, in Proc. IEEE International Conference on RFID. Dual-band channel gain statistics for dual-antenna tyre pressure monitoring RFID Tags, (2011), pp. 57-61, doi:10.1109/RFID.2011.5764637

22. JP Curty, M Declercq, C Dehollain, N Joehl, Design and, Optimization of Passive UHF RFID Systems. (Springer Science+Business Media, LLC, New York, 2007)

23. S Gabriel, R Lau, C Gabriel, The dielectric properties of biological tissue: III. parametric models for the dielectric spectrum of tissues. Phys. Med. Biol. 41(11), 2271-2293 (1996)

24. Impinj, Inc, Indy R1000 Reader Chip (IPJ-P1000) (2012). [http://www. impinj.com]

25. Impinj, Inc, Monza 5 Tag Chip Datasheet (IPJ-W1600) (2012). [http://www. impinj.com]

26. A Ahlbom, U Bergqvist, J Bernhardt, J Cesarini, L Court, M Grandolfo, M Hietanen, A Mckinlay, M Repacholi, D Sliney, J Stolwijk, M Swicord, L Szabo, M Taki, T Tenforde, H Jammet, R Matthes, Guidelines for limiting exposure to time-varying electric, magnetic, and electromagnetic fields (Up to $300 \mathrm{GHz}$ ). Health Phys. 74(4), 494-522 (1998)

27. IEEE standard for safety levels with respect to human exposure to radio frequency electromagnetic fields, $3 \mathrm{kHz}$ to $300 \mathrm{GHz}$. IEEE Std C95.1-1991 (1992)

doi:10.1186/1687-3963-2013-2

Cite this article as: Grosinger: Feasibility of backscatter RFID systems on the human body. EURASIP Journal on Embedded Systems 2013 2013:2.

\section{Submit your manuscript to a SpringerOpen ${ }^{\mathcal{O}}$ journal and benefit from:}

- Convenient online submission

- Rigorous peer review

- Immediate publication on acceptance

- Open access: articles freely available online

- High visibility within the field

- Retaining the copyright to your article

Submit your next manuscript at $\boldsymbol{\nabla}$ springeropen.com 